\title{
DETERMINATION OF CORRELATIONS BETWEEN SUBJECTS' PSYCHOPHYSIOLOGICAL PARAMETERS AND THE RESULTS OF THE PERCEPTUAL EXPERIMENT
}

\author{
Aleksey Grigorev, \& Viktor Gorodnyi \\ Department or Higher nervous activity and Psychophysiology, St Petersburg University (Russia)
}

\begin{abstract}
Nowadays in Russia, there are insufficient systematized data on the degree of preparedness of medical students and graduates to interact with children with atypical development (AD). We have developed a methodological approach to assess the interaction of medical students with AD children. The work uses a perceptual experiment during which students are presented with test sequences containing speech signals of children with typical development (TD) and AD (Down syndrome, autism spectrum disorders). During the perceptual experiment, the listeners' behavior was being video-recorded in parallel to verify their answers using the FaceReader software determining the true emotional state by their facial expression. The students' psycho-emotional status was evaluated using a battery of psychological tests before and after the perceptual experiment. Additionally, the subjects' psychophysiological and physiological parameters were determined. At the first stage of the work, the speech of $16 \mathrm{TD}$ and AD children (11 boys and 5 girls) was used, the listeners were 25 1st-year pediatric students and 5 experts in the field of child speech. The statistical analysis revealed correlations between the listeners' psychophysiological characteristics (Russian-native speakers): the profile of functional lateral asymmetry, formation indicators of the phonemic hearing, the coefficient of lateral preference by speech and the success in recognizing the children's gender, age, psychoneurological state (TD - developmental disorders), and the severity of disorders in AD children. The acoustic parameters of words classified by experts as "legible" and "illegible" were determined.

The work is financially supported by the Russian Science Foundation (project 19-78-00057).
\end{abstract}

Keywords: Perceptual experiment, atypical development, speech.

\section{Introduction}

The method of perceptual experiment is widely used to study various aspects of speech perception in adults and children, for example, to study the influence of the speaker's accent or fuzzy articulation on the perception of a speech message (Liu, Jaeger, 2018); to assess gender differences by voice features (Amir et al., 2012); to assess the speech development during ontogenesis in typical and atypical development (Lyakso, Frolova, \& Grigorev, 2016, 2017; Lyakso et al., 2019). Yet, there has been much less focus on identifying the correlations between the psychophysiological characteristics of listeners participating in the perceptual experiment and the success of recognizing various speaker's parameters (Lyakso et al., 2019). The goal of this study was to study the correlations between the listeners' psychophysiological characteristics and the success of assignments of the perceptual experiment.

The research is carried out within the framework of studies of the Child Speech Research Group of St Petersburg University aimed at studying the speech formation in ontogenesis in typical and atypical development.

\section{Materials and methods}

The research object is words cut from the speech of 5-7-year-old children with autism spectrum disorders (ASD), Down syndrome (DS), and typically developing (TD). The study recruited 11 boys and 5 girls. The speech material was selected from the "AD_Child.Ru" database (Lyakso, Frolova, \& Karpov, 2019), recordings were made under standardized conditions. To conduct the perceptual experiment, a test sequence containing 30 speech signals was created. Listeners were adult Russian-native speakers $(n=25$, age $18.2 \pm 0.9$ years; 4 men and 21 women), $1^{\text {st }}$-year students of the St Petersburg State Pediatric Medical 
University. The test sequence was presented in an open field. All the listeners were checked for hearing thresholds and the formation of phonemic hearing, the coefficient of lateral preference (CLP) by speech and the profile of functional lateral asymmetry (PFLA) were determined. The listeners were given a task to determine the child's gender, age, the presence or absence of developmental disorders, and, if any, the severity of disorders.

To identify correlations between the listeners' psychophysiological indicators and the success of their assignments of the perceptual experiment, statistical analysis was conducted in the STATISTICA 10.0 program using the Mann-Whitney U-test, correlation, regression, multiple regression, discriminant, and factor analyses.

Additionally, we conducted a study in which 5 experts took part ( 3 men and 2 women, age $29.2 \pm 6.5$ years, experience in the field of child speech $8.6 \pm 7.4$ years). The experts listened to the test sequence used in the previous study and determined the intelligibility of the speech signals. Spectrographic analysis of words identified by the experts as legible (with probability higher than 0.75) and illegible (the probability of determining as legible less than 0.25 ), regardless of which children pronounced them (TD, ASD, or DS), was carried out in the Cool Edit Pro 2.1 sound editor.

\section{Results}

The perceptual experiment showed that the listeners indicated the age of children close to the real one. According to median values, the age indicated by adults for children of all groups is 6 years old. When determining the speaker's gender, the listeners better recognize the male gender than the female one $(72.7 \%$ of correct answers and $32.7 \%$, respectively).

The correlation analysis (Spearman, $\mathrm{p}<0.05$ ) revealed a correlation between the listener's hearing thresholds (audiometry - left ear) and female gender recognition in TD children ( $\mathrm{r}=0.74)$, PFLA of listeners and male gender recognition in TD children $(0.70)$, the listeners' age and male gender recognition in children with DS (-0.53). The multiple regression analysis showed that the indicators of phonemic hearing are associated $\mathrm{F}(7,8)=1.5111 \mathrm{p}<0.28682$ with the male gender recognition in TD children (Beta $\left.=0.738 \mathrm{R}^{2}=0.569\right)$.

When determining the child's state, the listeners had to indicate the severity of developmental disorders: norm (TD) - mild disorders (ASD) - severe disorders (DS).

The listeners most often classified children in the appropriate group: children with typical development - in the group of norm, children with ASD - in the group of mild disorders, children with DS - in the group of severe disorders. The men determined TD children as children with typical development with probability of $55 \%$, children with ASD as having mild disorders with probability of $55 \%$, and children with DS as having severe disorders with probability of $65 \%$. The women determined TD children as children with typical development with probability of $61 \%$, children with ASD as having mild disorders with probability of $39 \%$, and children with DS as having severe disorders with probability of $66 \%$ (Figure 1).

Figure 1. Recognition of the psychoneurological state of children by listeners of the different gender. On the horizontal axis - children, on the vertical axis - the probability of recognition, \%. Black columns are data for men, white ones are for women. ${ }^{*} p<0.05$, Mann-Whitney test.

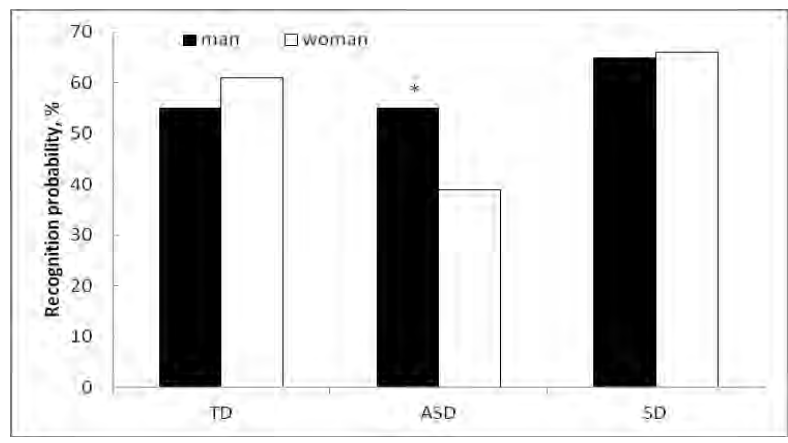

The data of regression analysis showed that the listeners' gender is associated $F(1,24)=4.8016$ $\mathrm{p}<0.03838$ with the state recognition in children with ASD (Beta $=-0.408 \mathrm{R}^{2}=0.167$ ). The correlation analysis (Spearman, $\mathrm{p}<0.05$ ) revealed a correlation between the listeners' gender and the state recognition in children with DS $(\mathrm{r}=0.32)$. The discriminant analysis found a correlation between the indicators of audiometry (left ear) $\mathrm{F}(3,2)=12.430 \mathrm{p}<0.0754$ and the state recognition in TD children (Wilks' Lambda=0.811). 
The factor analysis (factor rotation - Varimax raw, $\mathrm{p}=1.0$ ) of 10 variables: listener's gender, age, experience in communicating with children, psychophysiological indicators (phonemic hearing, audiometry (left and right ear), CLP by speech), state recognition in children TD, with DS, and with ASD, identified two factors with the following loadings. Factor 1 - the listener's age (-0.73), the listener's experience (-0.97), phonemic hearing (0.97), and state recognition in children with DS (0.75). Factor 2 - CLP by speech (0.99), audiometry indicators - left ear (0.73), and state recognition in children with ASD (0.87).

Using the instrumental analysis, the acoustic parameters of words determined by the experts as legible and illegible were identified. The duration of the legible words is $767.7 \pm 229.8 \mathrm{~ms}$; the duration of the illegible ones is $1105.3 \pm 276.3 \mathrm{~ms}$. The duration of the stressed vowel is $176.2 \pm 29.6 \mathrm{~ms}$ in the legible words and $346.6 \pm 237.5 \mathrm{~ms}$ in the illegible words. Thus, the words classified by the experts as legible are significantly shorter and have shorter stressed vowels. The pitch of the stressed vowels is $337.4 \pm 69.3 \mathrm{~Hz}$ in the legible words and $468.8 \pm 105.7 \mathrm{~Hz}$ in the illegible words. That is, the words classified by the experts as legible have significantly lower pitch values. There were no significant differences between the words classified by the experts as legible and illegible according to values of the difference between maximum and minimum pitch values, formant frequencies of stressed vowels, parameters reflecting the articulation clarity (formant triangles area and vowel articulation index).

\section{Conclusion}

The paper provides data on the influence of psychophysiological parameters of the listeners participants of the perceptual experiment on the success of assignments: the correlations between hearing thresholds, PFLA, the formation of phonemic hearing, listeners' age, and gender recognition of children were revealed. The parameters of listeners influencing the recognition of the psychoneurological state of children were determined: gender, age, experience, and the formation of phonemic hearing are associated with the state recognition in children with DS; gender, CLP by speech, and hearing thresholds with the state recognition in children with ASD; hearing thresholds with the state recognition in TD children. The acoustic parameters of words classified by the experts - specialists in the study of child speech, as legible and illegible have been determined. These categories of words differ in the parameters of the duration of words and stressed vowels and the pitch values of stressed vowels. This work is part of the research dedicated to identifying the ability of medical students to determine the presence/absence of developmental disorders in children and, if any, the severity of disorders.

\section{Acknowledgements}

The study is financially supported by the Russian Science Foundation (project 19-78-00057).

\section{References}

Amir O., Engel M., Shabtai E., Amir N. (2012). Identification of children's gender and age by listeners. $J$. Voice, 26, №. 3, 313-321.

Liu L., Jaeger T.F. (2018). Inferring causes during speech perception. Cognition, 174, 55-70.

Lyakso E., Frolova O., Gorodnyi V., Grigorev A., Nikolaev A., Matveev Yu. (2019). Reflection of the Emotional state in the characteristics of voice and speech of children with Down syndrome. SpeD 2019, The 10th Conference on Speech Technology and Human-Computer Dialog, October 10-12, Timisoara, Romania, 1-6.

Lyakso E., Frolova O., Grigorev A., Gorodnyi V., and Nikolaev A. (2019). Strategies of speech interaction between adults and preschool children with typical and atypical development. Behav. Sci, 9(12): 159.

Lyakso, E., Frolova, O., \& Grigorev, A. (2016). A comparison of acoustic features of speech of typically developing children and children with autism spectrum disorders. Lecture Notes in Computer Science, 9811, 43-50.

Lyakso, E., Frolova, O., \& Grigorev, A. (2017). Perception and acoustic features of speech of children with autism spectrum disorders. Lecture Notes in Computer Science, 10458, 602-612.

Lyakso, E., Frolova, O., \& Karpov, A. (2019). A new method for collection and annotation of speech data of atypically developing children. Proceedings of 2018 International Conference on Sensor Networks and Signal Processing, SNSP 2018, 175-180. 